Imperial/TP/2006/JG/04

hep-th/0611219

\title{
Properties of some conformal field theories with M-theory duals
}

\author{
Jerome P. Gauntlett ${ }^{1,2}$, Eoin Ó Colgáin ${ }^{1}$ and Oscar Varela ${ }^{3}$ \\ ${ }^{1}$ Theoretical Physics Group, Blackett Laboratory, \\ Imperial College, London SW' 2AZ, U.K. \\ ${ }^{2}$ The Institute for Mathematical Sciences, \\ Imperial College, London SW7 2PE, U.K. \\ ${ }^{3}$ Departamento de Física Teórica and IFIC, \\ Universidad de Valencia-CSIC, 46100-Burjassot (Valencia), Spain
}

\begin{abstract}
By studying classes of supersymmetric solutions of $D=11$ supergravity with $A d S_{5}$ factors, we determine some properties of the dual fourdimensional $N=1$ superconformal field theories. For some explicit solutions we calculate the central charges and also the conformal dimensions of certain chiral primary operators arising from wrapped membranes. For the most general class of solutions we show that there is a consistent Kaluza-Klein truncation to minimal $D=5$ gauged supergravity. This latter result allows us to study some aspects of the dual strongly coupled thermal plasma with a non-zero $R$-charge chemical potential and, in particular, we show that the ratio of the shear viscosity to the entropy density has the universal value of $1 / 4 \pi$.
\end{abstract}




\section{Introduction}

A classification of the most general supersymmetric solutions of $D=11$ supergravity that consist of a warped product of $A d S_{5}$ with a six-dimensional compact manifold $M_{6}$ was presented in [1]. Via the AdS/CFT correspondence these correspond to the most general class of four-dimensional conformal field theories (CFTs) with $N=1$ supersymmetry that have M-theory duals. It was shown that $M_{6}$ has a canonical $S U(2)$ structure that is related to the four-form flux and the warp factor, via some differential conditions. In the special sub-class where an almost complex structure on $M_{6}$ is assumed to be integrable, the differential conditions were integrated in [1] and a rich class of explicit solutions were constructed. For these explicit solutions the manifold $M_{6}$ is topologically an $S^{2}$ bundle over a four-dimensional base manifold $B_{4}$.

For the special case of explicit solutions where $B_{4}=S^{2} \times T^{2}$, after dimensional reduction and T-duality one obtains the $A d S_{5} \times Y^{p, q}$ solutions of type IIB string theory where $Y^{p, q}$ are an infinite class of Sasaki-Einstein metrics [1, 2]. For this class of solutions the dual conformal field theories were identified in [3] and this has led to many further developments. It would be desirable to have a similar understanding of the conformal field theories dual to the other explicit $A d S_{5} \times_{w} M_{6}$ solutions discovered in [1. Here, as a step in this direction, one of our goals will be to study the explicit solutions of [1 in more detail and extract some quantities of the dual CFT. We begin by determining the conditions imposed by quantisation of the four-form flux and then use this to calculate the central charges of the CFT. For the $B_{4}=S^{2} \times T^{2}$ case, we recover the known formula 2 for the central charge which has, for fixed $p$ and $q$, an $N^{2}$ scaling corresponding to the dual CFT being a gauge theory. For all other $B_{4}$ we find that the central charge scales like $N^{3}$, just as for the six-dimensional $(2,0)$ field theory that lives on M5-branes [4, indicating that the dual CFT is something exotic.

We next study supersymmetric probe membranes that wrap two-cycles in $M_{6}$, which correspond to chiral primary operators in the dual CFT. We first identify a generalised calibration [5] that can be constructed from the Killing spinors. We argue that if the membrane worldvolume is calibrated by the generalised calibration then it is supersymmetric. We determine the supersymmetric cycles for the explicit solutions and calculate the conformal dimensions of the chiral primaries finding that they all scale like $N$.

The final topic is a study of some aspects of the strongly coupled thermal plasma of the CFTs. Recall that the long distance, low-frequency behaviour of an interacting quantum field theory at finite temperature is expected to be well-described by fluid 
dynamics. Starting with the work of [6] there has been a number of papers that calculate various transport co-efficients, such as shear viscosity, $\eta$, and diffusion constants, for quantum field theories that have gravity duals. This is achieved by studying fluctuations about black hole geometries and employing the AdS/CFT correspondence. Following [6, 7, 8, 9] it was shown that, in the absence of chemical potentials, the ratio of shear viscosity to entropy density, $\eta / s$, is universal and equal to $1 / 4 \pi$ [10, 11]. For the case of $N=4$ super-Yang-Mills theory it was subsequently shown that this universal result persists in the presence of non-zero $R$-charge chemical potentials in [12, 13, 14] (a calculation for the theory living on M2-branes was performed in [15]). Most recently, it was shown in [16] that $\eta / s=1 / 4 \pi$ is also valid for the $N=1$ gauge theories that are dual to $A d S_{5} \times S E_{5}$ solutions of type IIB supergravity, where $S E_{5}$ is an arbitrary five-dimensional Sasaki-Einstein space.

Here we will show that for the CFTs dual to the most general supersymmetric $A d S_{5}$ solutions of [1] (i.e. not just the explicit solutions) we also have $\eta / s=1 / 4 \pi$. This is noteworthy since, as remarked above, the CFTs dual to these solutions must, in general, be quite different to the gauge theories that are dual to the $Y^{p, q}$ spaces. In addition we show that the speed of sound in the plasma is $1 / \sqrt{3}$.

Our approach for obtaining these results will follow that of [16]. It was shown in [16] that there is a consistent Kaluza-Klein truncation of type IIB supergravity on any $D=5$ Sasaki-Einstein space to minimal five-dimensional gauged supergravity. The gauge field of the gauged supergravity corresponds to the $R$-symmetry of the CFT. This means that any solution of the gauged supergravity will give rise to an exact solution of type IIB supergravity. This is remarkable since such consistent truncations are not common (see [17] for a relevant discussion). By studying some charged black hole solutions of the five-dimensional gauged supergravity, the authors of [16] then used the consistent truncation to extract results about the hydrodynamics of the thermal plasma of the dual CFT.

Here we will show that for the most general $A d S_{5} \times_{w} M_{6}$ solutions of [1] there is also a consistent truncation to five-dimensional minimal gauged supergravity. This result allows us to directly import some of the analysis of [16] to obtain the properties about the thermal plasma mentioned above.

The plan of the rest of the paper is as follows. Section 2 analyses flux quantisation and derives the central charges for some of the explicit solutions of [1]. Section 3 discusses supersymmetric wrapped membranes and, for the explicit solutions of section 2, we calculate the conformal dimension of the associated chiral primary operators. Section 4 discusses the consistent Kaluza-Klein truncation and this is 
used to normalise the $R$-charge of the wrapped membranes and also to study the hydrodynamics of the dual CFT. Section 5 briefly concludes.

\section{Flux quantisation and Central charges}

The class of supersymmetric solutions of $D=11$ supergravity that we shall consider in this paper were first analysed in [1]. The $D=11$ metric is a warped product

$$
d s^{2}=L^{2} e^{2 \lambda}\left[d s^{2}\left(A d S_{5}\right)+d s^{2}\left(M_{6}\right)\right]
$$

where $\lambda$ only depends on the coordinates of $M_{6}$. The metric $d s^{2}\left(A d S_{5}\right)$ is that of a unit radius $A d S_{5}$ and the length scale $L$ fixes the overall scale. The four-form flux $G_{(4)}$ is a four-form just on $M_{6}$. In general, it was shown that $M_{6}$ has an $S U(2)$ structure constructed from the Killing spinors that is specified by two one-forms $K^{1}$, $K^{2}$, a real two-form $J$, a complex two-form $\Omega$ and a scalar $\cos \zeta$. The metric on $M_{6}$ can be written

$$
d s^{2}\left(M_{6}\right)=e^{i} e^{i}+\left(K^{1}\right)^{2}+\left(K^{2}\right)^{2}
$$

with $J=e^{1} e^{2}+e^{3} e^{4}$ and $\Omega=\left(e^{1}+i e^{2}\right)\left(e^{3}+i e^{4}\right)$ where the products of forms are taken to be wedge products. The vector dual to $\cos \zeta K^{2}$ is a Killing vector which is related to the $R$-symmetry of the dual $\mathrm{CFT}$ as we shall discuss later. Introducing coordinates where this Killing vector is given by $3 \partial_{\psi}$ one can show that the metric can be written

$$
d s^{2}\left(M_{6}\right)=g_{i j}^{4} d x^{i} d x^{j}+e^{-6 \lambda} \sec ^{2} \zeta d y^{2}+\frac{1}{9} \cos ^{2} \zeta(d \psi+\rho)^{2},
$$

where $g_{i j}^{4}, \lambda, \zeta$ and $\rho$ are all functions of $x^{i}$ and $y$. In these coordinates we have $K^{1}=e^{-3 \lambda} \sec \zeta d y$ and $K^{2}=(1 / 3) \cos \zeta(d \psi+\rho)$. The expression for the most general four-form flux can be found in section 4 . The precise conditions that need to be imposed in order to get a supersymmetric solution, which include $2 y=e^{3 \lambda} \sin \zeta$, can be found in [1].

Some of this paper will focus on the explicit solutions of [1] which were constructed by demanding that the natural almost complex structure on $M_{6}$ is integrable and hence $M_{6}$ is a complex manifold. In this case, topologically, $M_{6}$ is an $S^{2}$ bundle over a four-dimensional base manifold $B_{4}$ that is either Kähler-Einstein with positive curvature, and hence $C P^{2}, S^{2} \times S^{2}$ or a del-Pezzo $d P_{k}, k=3, \ldots, 8$, or alternatively a product space $S^{2} \times S^{2}, S^{2} \times T^{2}$ or $S^{2} \times H^{2}$ (one can also replace $H^{2}$ with $H^{2} / \Gamma$ ). The $S^{2}$ fibration can be obtained by taking the canonical line-bundle of the fourdimensional Kähler base space and adding a "point at infinity" to each of the fibres. 
The detailed form of the metric on $M_{6}$ and the four-form for the explicit solutions of [1] that we shall study here will be given shortly.

In order to get a good solution of $M$-theory we need to ensure that the four-form flux is properly quantised. In particular we demand that

$$
N_{\Sigma_{4}} \equiv \frac{1}{(2 \pi)^{3} l_{11}^{3}} \int_{\Sigma_{4}} G_{(4)} \in \mathbb{Z}
$$

for any four-cycle $\Sigma_{4}$ on $M_{6}$, where $l_{11}$ is the $D=11$ Planck length. As usual, this leads to a quantisation condition on the length scale $L$.

The central charge of the dual conformal field theory can be obtained from the formula [18]

$$
c=\frac{\pi R_{A d S_{5}}^{3}}{8 G_{5}}
$$

where $G_{5}$ is the effective five-dimensional Newton constant and $R_{A d S_{5}}$ is the radius of $A d S_{5}$ in the five-dimensional theory. Taking the warp factor into account, a short calculation shows that

$$
c=\frac{1}{2^{7} \pi^{6}}\left(\frac{L}{l_{11}}\right)^{9} \int_{M_{6}} d^{6} x \sqrt{g\left(M_{6}\right)} e^{9 \lambda} .
$$

We now consider three explicit examples in turn. When $B_{4}$ is Kähler-Einstein, when $B_{4}=S^{2} \times S^{2}$ and when $B_{4}=T^{2} \times S^{2}$. The case $B_{4}=S^{2} \times H^{2}$ is very similar to the $S^{2} \times S^{2}$ case and for simplicity of presentation we omit the details.

\section{$2.1 \quad \mathrm{~B}_{4}=\mathrm{KE}_{4}$}

For this case the general solutions of [1] depend on two parameters $b$ and $c$. The internal metric is given by

$$
d s^{2}\left(M_{6}\right)=\frac{e^{-6 \lambda}}{3}\left(b-y^{2}\right) d s^{2}\left(K E_{4}\right)+e^{-6 \lambda} \sec ^{2} \zeta d y^{2}+\frac{1}{9} \cos ^{2} \zeta D \psi^{2},
$$

with

$$
\begin{aligned}
e^{6 \lambda} & =\frac{2\left(b-y^{2}\right)^{2}}{c y+2 b+2 y^{2}}, \\
\cos ^{2} \zeta & =\frac{-3 y^{4}-2 c y^{3}-6 b y^{2}+b^{2}}{\left(b-y^{2}\right)^{2}}
\end{aligned}
$$

and $d s^{2}\left(K E_{4}\right)$ is (a $y$ independent) four-dimensional Kähler-Einstein metric with positive curvature. We shall normalise this metric so that $\mathcal{R}=J_{K E}$ where $\mathcal{R}$ is the Ricci-form and $J_{K E}$ is the Kähler form. We also have $D \psi \equiv d \psi+P$ where $P$ is 
the connection on the canonical bundle of the $K E_{4}$, i.e. $d P=\mathcal{R}$. The $S^{2}$ fibre is parametrised by $y$ and $\psi$ : the coordinate $\psi$ is periodic with period $2 \pi$ and $y$ lies in an interval $y_{1} \leq y \leq y_{2}$ where the $y_{i}$ are appropriate roots of the quartic in the numerator of $\cos ^{2} \zeta$. The four-form flux is given by

$$
\frac{1}{L^{3}} G_{(4)}=\frac{4 y^{3}+3 c y^{2}+12 b y+b c}{18\left(y^{2}-b\right)} \operatorname{vol}_{K E_{4}}+\frac{y^{4}-6 b y^{2}-2 b c y-3 b^{2}}{9\left(y^{2}-b\right)^{2}} J_{K E} d y D \psi
$$

where the products of forms are taken to be wedge products.

In order to keep the analysis simple, we will now restrict to the $c=0$ class of solutions. For this class one can rescale to set $b=1$. The range of $y$ is now easily determined from $3 y^{4}+6 y^{2}-1=0$ : we take $y_{1} \leq y \leq y_{2}$ with

$$
y_{1,2}=\mp\left[\frac{2}{\sqrt{3}}-1\right]^{1 / 2} \text {. }
$$

To implement the flux quantisation condition (2.4) we need to identify a basis of four-cycles. If we let the two-cycles $\Sigma_{a}$ be a basis for $H_{2}\left(K E_{4}, \mathbb{Z}\right)$, then we can take the basis to be $C_{a}, C_{N}$ where $C_{a}$ are the four-cycles obtained by considering the $S^{2}$ fibration over the two-cycles $\Sigma_{a}$ and $C_{N}$ is the four-cycle obtained by restricting $y$ to lie at the "north pole" of the two-sphere fibre by setting $y=y_{2}$. Instead of $C_{N}$ we could also consider the four-cycle $C_{S}$ sitting at the "south pole" of the fibre by setting $y=y_{1}$.

It will be useful to introduce some notation concerning the $K E_{4}$ base space. In particular we have

$$
n\left(\Sigma_{a}\right)=\frac{1}{2 \pi} \int_{\Sigma_{a}} \mathcal{R}=m n_{a}
$$

where the positive integer $m$ is known as the Fano index of $K E_{4}$ and is the largest positive integer such that all of the $n_{a}$ are integers. We also define

$$
M=\frac{1}{4 \pi^{2}} \int_{K E_{4}} \mathcal{R} \wedge \mathcal{R}
$$

and we note that $M$ is always divisible by $m^{2}$. For further discussion see e.g. appendix B of [19], but we note here that the explicit values for $(m, M)$ for $C P^{2}$ are $(3,9)$, for $S^{2} \times S^{2}$ are $(2,8)$ and for $d P_{k}, k=3, \ldots, 8$ are $(1,9-k)$.

We now calculate the flux threading through the various cycles. Using (2.4), we find

$$
\begin{aligned}
& N_{C_{N}}=-\left(\frac{y_{2} L^{3}(2+\sqrt{3})}{18 \pi l_{11}^{3}}\right) M \\
& N_{C_{a}}=-\left(\frac{y_{2} L^{3}(2+\sqrt{3})}{18 \pi l_{11}^{3}}\right) 2 m n_{a} .
\end{aligned}
$$


We note that $N_{C_{S}}=-N_{C_{N}}$. In order to ensure that $N_{C_{N}}$ and $N_{C_{a}}$ are indeed integers, we choose the length scale $L$ to be given by

$$
\frac{y_{2} L^{3}(2+\sqrt{3})}{18 \pi l_{11}^{3}}=\frac{N}{h},
$$

where $N$ is an arbitrary integer and $h$ is the highest common factor of $M$ and $2 m$. We then have $N_{C_{N}}=-(M / h) N$ and $N_{C_{a}}=-(2 m / h) n_{a} N$.

Using (2.6), we are now in a position to determine the central charge in terms of these brane charges and we find

$$
c=9(3 \sqrt{3}-5) \frac{M}{h^{3}} N^{3} .
$$

\section{$2.2 \quad \mathrm{~B}_{4}=\mathrm{S}^{2} \times \mathrm{S}^{2}:$}

When $B_{4}=S^{2} \times S^{2}$ the general solutions of [1] depend on three parameters $a_{1}, a_{2}$ and $c$. The metric takes the form

$$
\begin{aligned}
d s^{2}\left(M_{6}\right) & =\frac{e^{-6 \lambda}}{3}\left(a_{1}-y^{2}\right) d s^{2}\left(S_{(1)}^{2}\right)+\frac{e^{-6 \lambda}}{3}\left(a_{2}-y^{2}\right) d s^{2}\left(S_{(2)}^{2}\right) \\
& +e^{-6 \lambda} \sec ^{2} \zeta d y^{2}+\frac{1}{9} \cos ^{2} \zeta D \psi^{2}
\end{aligned}
$$

where $d s^{2}\left(S_{(1)}^{2}\right)$ and $d s^{2}\left(S_{(2)}^{2}\right)$ are both canonical unit radius metrics on two-spheres. Also, again, $D \psi=d \psi+P$ with $0 \leq \psi \leq 2 \pi$ and now $d P=\operatorname{vol}\left(S_{(1)}^{2}\right)+\operatorname{vol}\left(S_{(2)}^{2}\right)$. We also have

$$
\begin{aligned}
e^{6 \lambda} & =\frac{2\left(y^{2}-a_{1}\right)\left(y^{2}-a_{2}\right)}{2 y^{2}+c y+a_{1}+a_{2}} \\
\cos ^{2} \zeta & =\frac{-3 y^{4}-2 c y^{3}-3\left(a_{1}+a_{2}\right) y^{2}+a_{1} a_{2}}{\left(y^{2}-a_{1}\right)\left(y^{2}-a_{2}\right)}
\end{aligned}
$$

The four-form flux is given by

$$
\begin{aligned}
\frac{1}{L^{3}} G_{(4)}= & \frac{1}{18\left(y^{2}-a_{1}\right)\left(y^{2}-a_{2}\right)}\left[4 y^{5}+3 c y^{4}+4 y^{3}\left(a_{1}+a_{2}\right)-c y^{2}\left(a_{1}+a_{2}\right)\right. \\
& +\frac{\left.-2 y\left(a_{1}^{2}+a_{2}^{2}+4 a_{1} a_{2}\right)-c a_{1} a_{2}\right] \operatorname{vol}\left(S_{(1)}^{2}\right) \operatorname{vol}\left(S_{(2)}^{2}\right)}{9\left(a_{2}^{2}-a_{1}\right)^{2}} \operatorname{vol}\left(S_{(2)}^{2}\right) d y D \psi \\
& +\frac{\left[y^{4}-y^{2}\left(a_{1}+5 a_{2}\right)-2 a_{2} c y-a_{1} a_{2}-2 a_{2}^{2}\right]}{9\left(y^{2}-a_{2}\right)^{2}} \operatorname{vol}\left(S_{(1)}^{2}\right) d y D \psi .
\end{aligned}
$$

Observe that when $a_{1}=a_{2}=a$ the solution is the same as the Kähler-Einstein class when $K E_{4}$ is taken to be $S^{2} \times S^{2}$. 
For simplicity, in this section we shall again restrict to the $c=0$ class of solutions. After a rescaling the solution only depends on the ratio $z=a_{1} / a_{2}$. Without loss of generality we can set

$$
a_{1}=z, \quad a_{2}=1
$$

and take $0<z \leq 1$. The range of $y$ is now easily determined from the zeroes of $\cos ^{2} \zeta$ and we find

$$
y_{1,2}=\mp\left[-\frac{1}{2}(z+1)+\frac{1}{6} \sqrt{X}\right]^{1 / 2} .
$$

where $X=9 z^{2}+30 z+9$.

To implement the flux quantisation we will consider the flux through the cycles $C_{1}, C_{2}$, which are the obtained by considering the $S^{2}$ fibration over $S_{(1)}^{2}$ (at a fixed point on $S_{(2)}^{2}$ ) and $S_{(2)}^{2}$ (at a fixed point on $S_{(1)}^{2}$ ), respectively, and $C_{N}$, which is the four-cycle sitting at the north pole, $y=y_{2}$, of the two-sphere fibre. We find

$$
\begin{aligned}
& N_{C_{1}}=-\frac{y_{2} L^{3}}{18 \pi l_{11}^{3}}[5+3 z+\sqrt{X}], \\
& N_{C_{2}}=-\frac{y_{2} L^{3}}{18 \pi l_{11}^{3}} \frac{[5 z+3+\sqrt{X}]}{z}, \\
& N_{C_{N}}=-\frac{y_{2} L^{3}}{18 \pi l_{11}^{3}} \frac{[X+3(1+z) \sqrt{X}]}{3 z} .
\end{aligned}
$$

Note that only two of the above charges are independent since $N_{C_{1}}+N_{C_{2}}=N_{C_{N}}$. Taking the ratio of (2.22) to (2.24) we get the expression

$$
\frac{N_{C_{2}}}{N_{C_{1}}}=\frac{1}{z} \frac{[(5 z+3)+\sqrt{X}]}{[(5+3 z)+\sqrt{X}]},
$$

which can be made a rational number $w$ by choosing $z$ such that

$$
z=\frac{2-w+2 w^{2}-2 \sqrt{1-w-w^{3}+w^{4}}}{3 w} .
$$

The range of $z \in(0,1]$ is covered once if we impose $w \in[1, \infty)$. Let us write $w=p / q$ for positive integers $p$ and $q$ with no common factors and $p>q$ (we also allow $p=q=1$ ) and hence

$$
z=\frac{2 q^{2}-q p+2 p^{2}-2(p-q) \sqrt{q^{2}+q p+p^{2}}}{3 p q} .
$$

We then choose the length scale $L$ via

$$
\frac{y_{2} L^{3}}{18 \pi l_{11}^{3}}[5+3 z+\sqrt{X}]=q N .
$$


This implies that $N_{C_{1}}=-q N, N_{C_{2}}=-p N$ and $N_{C_{N}}=-(p+q) N$ are indeed all integers.

The central charge can now be calculated and we find that it can be written as

$$
c=\frac{3^{3 / 2}}{2^{6}} \frac{\left[9(z+1)^{3}-\left(3 z^{2}+4 z+3\right) \sqrt{X}\right]}{z^{3 / 2}}(p q)^{3 / 2} N^{3} .
$$

As a check on this result, we should be in a position to rederive the earlier central charge for the $K E_{4}$ base in the special case where the base is $S^{2} \times S^{2}$. Setting $z=1$ $(p=q=1), m=2$ and $M=8$ we do indeed find agreement.

\section{$2.3 \quad \mathrm{~B}_{4}=\mathrm{S}^{2} \times \mathrm{T}^{2}$ and $\mathrm{Y}^{\mathrm{p}, \mathrm{q}}$ :}

We shall now consider the case when the base space $B_{4}=T^{2} \times S^{2}$. Recall that after dimensional reduction and T-duality, these solutions gave rise to the $A d S_{5} \times Y^{p, q}$ solutions of type IIB string theory where $Y^{p, q}$ are Sasaki-Einstein spaces. The analysis of [2] of the regularity of the $Y^{p, q}$ spaces and the quantisation of the five-form flux can be translated into the M-theory setting. We now present some of the details.

The metric is given by

$$
d s^{2}\left(M_{6}\right)=e^{-6 \lambda} d s^{2}\left(T^{2}\right)+\frac{1-c y}{6} d s^{2}\left(S^{2}\right)+e^{-6 \lambda} \sec ^{2} \zeta d y^{2}+\frac{1}{9} \cos ^{2} \zeta D \psi^{2},
$$

where $d s^{2}\left(S^{2}\right)$ is the metric on a unit radius two-sphere and $d s^{2}\left(T^{2}\right)$ is a metric on a two-torus. As above, $\psi$ has period $2 \pi$ and $D \psi=d \psi+P$ now with $d P=\operatorname{vol}\left(S^{2}\right)$. We also have

$$
\begin{aligned}
e^{6 \lambda} & =\frac{2\left(a-y^{2}\right)}{1-c y} \\
\cos ^{2} \zeta & =\frac{a-3 y^{2}+2 c y^{3}}{a-y^{2}}
\end{aligned}
$$

where $a \in(0,1)$ is constant. Here we will only consider $c \neq 0$ and so without loss of generality we can set $c=1$. The range of $y$ is given by $y_{1} \leq y \leq y_{2}$ where $y_{1}$ and $y_{2}$ are the smallest roots of the cubic $a-3 y^{2}+2 y^{3}=0$.

The four-form flux is given by

$$
\begin{aligned}
\frac{1}{L^{3}} G_{(4)} & =\frac{-2 y+y^{2}+a}{6\left(a-y^{2}\right)} \operatorname{vol}\left(S^{2}\right) \operatorname{vol}\left(T^{2}\right)-\frac{2(1-y)}{9} d y \operatorname{D} \psi \operatorname{vol}\left(S^{2}\right) \\
& -\frac{a+y^{2}-2 a y}{3\left(a-y^{2}\right)^{2}} d y \operatorname{D} \psi \operatorname{vol}\left(T^{2}\right)
\end{aligned}
$$


It was shown in [2] that the parameter $a$ is fixed in terms of two relatively prime integers $p$ and $q, p>q>0$ via:

$$
a=\frac{1}{2}+\frac{3 q^{2}-p^{2}}{4 p^{3}} \sqrt{4 p^{2}-3 q^{2}}
$$

The explicit expressions for the roots $y_{i}$ are then given by

$$
\begin{aligned}
y_{1} & =\frac{1}{4 p}\left(2 p-3 q-\sqrt{4 p^{2}-3 q^{2}}\right), \\
y_{2} & =\frac{1}{4 p}\left(2 p+3 q-\sqrt{4 p^{2}-3 q^{2}}\right) .
\end{aligned}
$$

The $Y^{p, q}$ spaces are $U(1)$ bundles over a four-dimensional base-space with Chernnumbers given by $p$ and $q$. The size of the $S^{1}$ fibre is $2 \pi l$, which means that the size of the M-theory two-torus is $4 \pi^{2}\left(l_{11} / L\right)^{3} / l$ (see, e.g., section 6 of [19]). Furthermore, the overall length scale of the type IIB solution translates into the parameter $L$ of the $D=11$ solution taking the form

$$
\frac{L^{3}}{12 \pi l_{11}^{3}}=\frac{p^{2}}{q\left[2 p+\sqrt{4 p^{2}-3 q^{2}}\right]} N,
$$

where $N$ is an arbitrary integer. Using this we can write

$$
\operatorname{vol}\left(T^{2}\right)=\pi \frac{q^{2}\left[p+\sqrt{4 p^{2}-3 q^{2}}\right]}{p^{2} N} .
$$

We now confirm that these constraints ensure that the four-form flux is properly quantised. If we let $C_{S^{2}}$ and $C_{T^{2}}$ denote the four-cycles given by the $S^{2}$ fibration over the $S^{2}$ and $T^{2}$, respectively, and $C_{N}$ the four-cycle given by the north pole of the fibration, $y=y_{2}$, we find

$$
\begin{aligned}
N_{C_{S^{2}}} & =-N, \\
N_{C_{T^{2}}} & =-p, \\
N_{C_{N}} & =q-p .
\end{aligned}
$$

In addition we have $N_{C_{S}}=(q+p)$. To compare with the IIB results, one should choose a different basis of four-cycles. In particular, instead of $C_{T^{2}}$ and $C_{N}$ we choose $(1 / 2)\left(C_{S}-C_{N}\right)$ and $(1 / 2)\left(C_{S}+C_{N}\right)$ (these are the four-cycles $C_{1} \times T^{2}$ and $C_{2} \times T^{2}$ where the $C_{i}$ are the basis of two-cycles on the $S^{2}$ fibration over $S^{2}$ that were discussed in [2]). We find that the corresponding fluxes are given by $p$ and $q$, respectively. 
Calculating the central charge using (2.6) we get

$$
\begin{aligned}
c & =\frac{3 p^{2}\left[3 q^{2}-2 p^{2}+p \sqrt{4 p^{2}-3 q^{2}}\right]}{4 q^{2}\left[2 p+\sqrt{4 p^{2}-3 q^{2}}\right]} N^{2} \\
& =\frac{\pi^{3} N^{2}}{4 \operatorname{Vol}\left(Y^{p, q}\right)},
\end{aligned}
$$

in agreement with [2] as expected. Note that in contrast to the $B_{4}$ considered in the last two subsections, when $B_{4}=S^{2} \times T^{2}$, not all of the fluxes scale with $N$; this leads

to the $N^{2}$ scaling behaviour for the central charge with fixed $p$ and $q$, rather than the $N^{3}$ behaviour we saw previously.

\section{Supersymmetric Membranes and Chiral Primaries}

We now consider probe membranes wrapping supersymmetric cycles on $M_{6}$. These configurations correspond to chiral primaries in the dual conformal field theories. For the most general class of solutions of [1] we claim that the conformal dimension of these operators can be obtained from the formula

$$
\Delta\left(\Sigma_{2}\right)=\tau_{M 2} L^{3} \int_{\Sigma_{2}} e^{3 \lambda} \operatorname{vol}_{M_{6}}\left(\Sigma_{2}\right) .
$$

Here $\operatorname{vol}_{M_{6}}\left(\Sigma_{2}\right)$ is the volume form of the cycle induced from the metric $d s^{2}\left(M_{6}\right)$ and $\tau_{M 2}$ is the tension of the membrane and is given in our conventions by $\tau_{M 2}=$ $1 /\left(4 \pi^{2} l_{11}^{3}\right)$. That the volume of the wrapped brane (suitably dressed by the warp factor) should be associated with the conformal dimension of the operators rather than the mass of the operators was explained in the context of Sasaki-Einstein compactifications in type IIB supergravity in [20. In the next section we will confirm that this is indeed the correct formula, by showing that the conformal dimension is related to the $R$-charge of these operators by the correct formula, $\Delta=(3 / 2) R$.

In order to be supersymmetric, the membranes must wrap two-cycles in $M_{6}$ that are calibrated by a certain two-form (generalised) calibration [5]. Recall [1] that the $D=11$ Killing spinors of the most general solutions (2.1) have the form

$$
\epsilon=\psi \otimes e^{\lambda / 2} \xi,
$$

where $\psi$ is a Killing spinor on $A d S_{5}$ and $\xi$ is a non-chiral spinor on $M_{6}$. The $S U(2)$ structure specified by $J, \Omega, K^{1}, K^{2}$ and $\cos \zeta$ can be constructed from bi-linears of $\xi$ as explained in [1]. Continuing to use the notation of [1], let us focus on the bi-linear 
two-form given by

$$
\begin{aligned}
Y^{\prime} & =\frac{1}{2} \bar{\xi} \gamma_{(2)} \gamma_{7} \xi \\
& =-\sin \zeta J+K^{1} \wedge K^{2}
\end{aligned}
$$

Using the results of [1] a short calculation now shows that $Y^{\prime}$ always satisfies the generalised calibration condition

$$
d\left(e^{3 \lambda} Y^{\prime}\right)=-i_{\tilde{K}^{2}} \frac{G_{(4)}}{L^{3}}
$$

where $\tilde{K}^{2}=\cos \zeta K^{2}$ is the one-form whose dual vector on $M_{6}$ is Killing (in the coordinates in (2.3) it is $\tilde{K}^{2}=3 \partial_{\psi}$ ). We now claim that if a membrane wraps a two-cycle $\Sigma_{2}$ on $M_{6}$ which is calibrated by $Y^{\prime}$, i.e.

$$
\operatorname{vol}_{M_{6}}\left(\Sigma_{2}\right)=\left.Y^{\prime}\right|_{\Sigma_{2}}
$$

then it is a supersymmetric configuration.

One way to see why $Y^{\prime}$ is the relevant object is to consider the two-form $\bar{\Omega}$, the membrane generalised calibration, of the full $D=11$ solution. Recall that for any supersymmetric solution of $D=11$ supergravity with a Killing spinor $\epsilon$ one can construct the bilinears $\bar{K}$, a one-form, and $\bar{\Omega}$, a two-form (there is also a five-form that is not relevant for the present discussion). It was shown in [21] that $\bar{\Omega}$ satisfies the generalised calibration condition $d \bar{\Omega}=i_{\bar{K}} G_{(4)}$. Next, restricting to the class of supersymmetric $A d S_{5}$ solutions of interest, we can use the decomposition of the $D=11$ Killing spinor (3.2) to show that restricting to directions tangent to $M_{6}$, we have $\bar{\Omega} \rightarrow 2 e^{\lambda} \bar{\psi} \psi Y^{\prime}$ and $\bar{K} \rightarrow-2 e^{\lambda} \bar{\psi} \psi \tilde{K}^{2}$. Properly taking into account the conformal factors then leads to (3.4).

We shall now give detailed expressions for the calibration two-form $Y^{\prime}$ (3.3) for the explicit solutions of [1] that we considered in the last section, and then use this to elucidate the corresponding calibrated cycles satisfying (3.5). As an extra check that we have indeed properly identified supersymmetric cycles, we have carried out a direct analysis of the $\kappa$-symmetry conditions in appendix A (similar calculations have been carried out in the context of Sasaki-Einstein solutions in [22, 23]). Having identified the supersymmetric cycles, the dimension of the corresponding chiral operators is determined using (3.1). 


\section{$3.1 \quad \mathrm{~B}_{4}=\mathrm{KE}_{4}$ :}

For this case the calibration two-form (3.3) is given by

$$
Y^{\prime}=-\frac{e^{-6 \lambda}}{3}\left(b-y^{2}\right) \sin \zeta J_{K E}+\frac{e^{-3 \lambda}}{3} d y D \psi .
$$

We now consider a membrane wrapping the two sphere fibre at a fixed point on the $K E_{4}$. From (2.7) the volume form for this cycle is simply $\left(e^{-3 \lambda} / 3\right) d y d \psi$ and hence it is calibrated by $Y^{\prime}$. The dimension of the dual chiral primaries is given by (3.1) and, for the class of solutions with $c=0, b=1$ for which we carried out the flux quantisation in the last section, we find

$$
\Delta=6(2-\sqrt{3}) \frac{N}{h} .
$$

We can also consider a membrane wrapping a two-cycle on $K E_{4}$ sitting at one of the poles of the $S^{2}$ fibration, $y=y_{1}, y_{2}$. Since at the poles we have $\sin \zeta= \pm 1$ it is clear that a holomorphic two-cycle $C_{i}$ on $K E_{4}$, i.e. one that is calibrated by $J_{K E}$, at $y=y_{1}, y_{2}$ is calibrated by $Y^{\prime}$. We can expand such a cycle in the basis $\Sigma_{a}$ of $H_{2}\left(K E_{4}, \mathbb{Z}\right)$ that we introduced in section 2.1 as $C_{i}=C_{i}^{a} \Sigma_{a}$ where $C_{i}^{a} \in \mathbb{Z}$. For membranes wrapping these cycles, in the case that $c=0, b=1$, we then find that

$$
\Delta=3(\sqrt{3}-1) \frac{C_{i}^{a} m n_{a}}{h} N .
$$

The fact that the $\Delta$ is the same for either pole is because $y_{2}=-y_{1}$.

\section{$3.2 \quad \mathrm{~B}_{4}=\mathrm{S}^{2} \times \mathrm{S}^{2}:$}

For this case we have

$$
Y^{\prime}=-\frac{e^{-6 \lambda}}{3}\left(a_{1}-y^{2}\right) \sin \zeta \operatorname{vol}\left(S_{(1)}^{2}\right)-\frac{e^{-6 \lambda}}{3}\left(a_{2}-y^{2}\right) \sin \zeta \operatorname{vol}\left(S_{(2)}^{2}\right)+\frac{e^{-3 \lambda}}{3} d y D \psi .
$$

The two-sphere fibre is again calibrated by $Y^{\prime}$. When $c=0, a_{1}=z, a_{2}=1$, membranes wrapping this cycle gives rise to chiral primaries with

$$
\Delta=\frac{3 q}{8}(5+3 z-\sqrt{X}) N .
$$

In addition the two-spheres on the base, $S_{(1)}^{2}$ or $S_{(2)}^{2}$, located at either $y=y_{1}$ or $y=y_{2}$ are also calibrated by $Y^{\prime}$. The conformal dimensions corresponding to the two-cycles on the base at either pole are the same and are given, when $c=0, a_{1}=z, a_{2}=1$, by

$$
\begin{aligned}
\Delta\left(S_{(1)}^{2}\right) & =\frac{3 q}{8}(-3 z-1+\sqrt{X}) N \\
\Delta\left(S_{(2)}^{2}\right) & =\frac{3 q}{16 z}\left(-3 z^{2}-8 z+3+(1+z) \sqrt{X}\right) N
\end{aligned}
$$




\section{$3.3 \quad \mathrm{~B}_{4}=\mathrm{S}^{2} \times \mathrm{T}^{2}$ and $\mathrm{Y}^{\mathrm{p}, \mathrm{q}}$ :}

This case provides a good check on our formulae since we can compare with the dimension of the chiral primaries that have already been calculated in the context of type IIB supergravity using the Sasaki-Einstein spaces $Y^{p, q}$. For this case we find that the calibration two-form is given by

$$
Y^{\prime}=-\frac{e^{-6 \lambda}}{3}\left(a-y^{2}\right) \sin \zeta \operatorname{vol}\left(S^{2}\right)-e^{-6 \lambda} \sin \zeta \operatorname{vol}\left(T^{2}\right)+\frac{e^{-3 \lambda}}{3} d y D \psi
$$

The two-sphere fibre is once again calibrated by $Y^{\prime}$ and the corresponding conformal dimension is given by

$$
\Delta=\frac{p}{q^{2}}\left(2 p-\sqrt{4 p^{2}-3 q^{2}}\right) N
$$

After dimensional reduction and $T$-duality the wrapped membrane corresponds to a D3-brane wrapping the three-cycle given by fixing a point on the round two-sphere in the $Y^{p, q}$ space. Our result for $\Delta$ agrees with that given in [24].

We next consider membranes wrapping the two-sphere by fixing a point on the $T^{2}$ and fixing $y=y_{1}$ or $y=y_{2}$. These are calibrated by $Y^{\prime}$ and we find

$$
\begin{aligned}
& \Delta\left(S^{2}\left(y_{1}\right)\right)=\frac{1}{2 q^{2}}\left(-4 p^{2}+2 p q+3 q^{2}+(2 p-q) \sqrt{4 p^{2}-3 q^{2}}\right) N \\
& \Delta\left(S^{2}\left(y_{2}\right)\right)=\frac{1}{2 q^{2}}\left(-4 p^{2}-2 p q+3 q^{2}+(2 p+q) \sqrt{4 p^{2}-3 q^{2}}\right) N .
\end{aligned}
$$

After dimensional reduction and $T$-duality these wrapped membranes correspond to D3-branes wrapping three-cycles in $Y^{p, q}$ that were discussed in [25] and our formula for $\Delta$ is in exact agreement.

We can also consider membranes wrapping the $T^{2}$ by fixing a point on the $S^{2}$ and fixing $y=y_{1}$ or $y=y_{2}$. These are also calibrated by $Y^{\prime}$ and we find

$$
\begin{aligned}
\Delta\left(T^{2}\left(y_{1}\right)\right) & =\frac{p}{2 q}\left(3 q+2 p-\sqrt{4 p^{2}-3 q^{2}}\right), \\
\Delta\left(T^{2}\left(y_{2}\right)\right) & =\frac{p}{2 q}\left(3 q-2 p+\sqrt{4 p^{2}-3 q^{2}}\right) .
\end{aligned}
$$

After dimensional reduction and $T$-duality these wrapped membranes correspond to momentum waves around the $\alpha$ direction in the Sasaki-Einstein picture. In fact these are precisely the conformal dimensions of the long BPS mesonic operators $\mathcal{L}_{ \pm}$ of the dual CFT that were noted in [26] where they were also identified with massless geodesics in the $A d S_{5} \times Y^{p, q}$ solutions. 


\section{Consistent Truncation}

In this section we show that for the most general class of supersymmetric $A d S_{5}$ solutions of [1] there is a consistent truncation to minimal five-dimensional gauged supergravity. We shall argue that the abelian gauge field of the gauged supergravity theory can be identified with the $R$-symmetry of the dual SCFT. We use this to determine the $R$-charges of the chiral primaries dual to the wrapped branes discussed in the last section and demanding that $\Delta=3 / 2 R$ we derive the formula for $\Delta$ presented in (3.1). Following this we discuss some aspects of the fluid-dynamics of the thermal plasma of the SCFTs with non-zero chemical potential.

\subsection{The ansatz}

The ansatz for the $D=11$ metric is given by (2.1) where we replace the $A d S_{5}$ metric with an arbitrary $D=5$ metric:

$$
d s^{2}=L^{2} e^{2 \lambda}\left[d s_{5}^{2}+d s^{2}\left(M_{6}\right)\right]
$$

with

$$
\begin{aligned}
d s^{2}\left(M_{6}\right) & =g_{i j}^{4}(x, y) d x^{i} d x^{j}+e^{-6 \lambda} \sec ^{2} \zeta d y^{2}+\frac{1}{9} \cos ^{2} \zeta(d \psi+\rho+A) \\
& =e^{i} e^{i}+\left(e^{5}\right)^{2}+\left(e^{6}\right)^{2}
\end{aligned}
$$

where $e^{5} \equiv K^{1}=e^{-3 \lambda} \sec \zeta$ and $e^{6}=K^{2}+(1 / 3) \cos \zeta A$ and $i, j=1, \ldots, 4$. Here we have just made the shift $d \psi \rightarrow d \psi+A$ in the general metric (2.3) of [1].

The ansatz for the four-form is much less obvious. After some trial and error we find that it is given by

$$
\frac{1}{L^{3}} G_{(4)}=d \bar{C}+\frac{1}{3} d y\left[\left(*_{5} F\right)+\frac{1}{3} A F\right]
$$

where

$$
\bar{C}=C^{0}+\frac{1}{3} A\left(e^{3 \lambda} Y^{\prime}\right)
$$

and $L^{3} d C^{0}$ is the four-form flux of the undeformed solution of [1] given below. After a little reorganisation, one finds that

$$
\frac{1}{L^{3}} G_{(4)}=g+\frac{e^{3 \lambda}}{3}\left(-\sin \zeta J+K^{1} e^{6}\right) F+\frac{1}{3} e^{3 \lambda} \cos \zeta K^{1}\left(*_{5} F\right)
$$

where $g$ is the four-form flux given in [1] after the substitution $d \psi \rightarrow d \psi+A$ :

$$
\begin{aligned}
g= & -\frac{1}{2} e^{12 \lambda} \partial_{y}\left(e^{-6 \lambda}\right) J^{2}-e^{-3 \lambda} \sec \zeta\left(*_{4} d_{4} e^{6 \lambda}\right) K^{1} \\
& -\frac{1}{3} e^{6 \lambda} \cos ^{3} \zeta\left(*_{4} \partial_{y} \rho\right) e^{6}+e^{3 \lambda}\left[\frac{1}{3} \cos ^{2} \zeta\left(*_{4} d_{4} \rho\right)-4 J\right] K^{1} e^{6}
\end{aligned}
$$


We now substitute this ansatz into the $D=11$ equations of motion:

$$
\begin{gathered}
R_{\mu \nu}-\frac{1}{12}\left(G_{\mu \sigma_{1} \sigma_{2} \sigma_{3}} G_{\nu}{ }^{\sigma_{1} \sigma_{2} \sigma_{3}}-\frac{1}{12} g_{\mu \nu} G^{2}\right)=0, \\
d G_{(4)}=0, \quad d * G_{(4)}+\frac{1}{2} G_{(4)} \wedge G_{(4)}=0,
\end{gathered}
$$

where $G^{2}=G_{\sigma_{1} \sigma_{2} \sigma_{3} \sigma_{4}} G^{\sigma_{1} \sigma_{2} \sigma_{3} \sigma_{4}}$. After some calculation! 1 using the conditions in [1], we deduce that the $D=5$ metric $g$ and the two-form field strength $F=d A$ must satisfy

$$
\begin{array}{r}
R_{a b}=-4 g_{a b}+\frac{1}{6}\left[F_{a c} F_{b}^{c}-\frac{1}{6} g_{a b} F^{2}\right], \\
d\left(*_{5} F\right)+\frac{1}{3} F \wedge F=0,
\end{array}
$$

where $F^{2}=F_{a b} F^{a b}$. These equations can be derived from an action whose lagrangian is given by

$$
\sqrt{-g}\left[R+12-\frac{1}{12} F^{2}+\frac{1}{108} \epsilon^{a_{1} a_{2} a_{3} a_{4} a_{5}} F_{a_{1} a_{2}} F_{a_{3} a_{4}} A_{a_{5}}\right]
$$

which is precisely the bosonic Lagrangian of minimal $D=5$ gauged supergravity.

We have thus shown that our ansatz is a consistent Kaluza-Klein truncation at the level of bosonic fields. In particular, any bosonic solution of minimal $D=5$ gauged supergravity can be uplifted using the ansatz to give a solution of $D=11$ supergravity that is based on an arbitrary general supersymmetric $A d S_{5}$ solution of [1. In appendix B we show that the Kaluza-Klein truncation is also consistent after including the fermions.

\section{$4.2 \quad R$-charge}

Recall that the Killing vector $\partial_{\psi}$ arises in all supersymmetric $A d S_{5}$ solutions of $D=11$ supergravity and hence it must be related to the $R$-symmetry in the dual SCFT. Clearly the ansatz for consistent truncation above involves gauging shifts of the coordinate $\psi$ and so it is natural to identify the gauge field $A$ with the $R$-symmetry.

While we claim that this is indeed the right interpretation it is worth mentioning a subtlety. In general the $R$-symmetry should be related to a linear combination of the isometry generated by the Killing vector $\partial_{\psi}$ with gauge transformations of the

\footnotetext{
${ }^{1}$ The Bianchi identity for $G_{4}$ is simple to verify. In checking the equations of motion for $G_{4}$ we used equations (2.47) and (2.49) of [1] and we also used the exterior derivative of $e^{6 \lambda}$ times equation (2.16) of [1]. In checking the Einstein equations we used the fact that the $A d S_{5}$ is a solution and focused on the $F$ dependent pieces.
} 
$D=11$ three-form potential $C_{(3)}$ which satisfies $d C_{(3)}=G_{(4)}$. This can be seen in detail in the context of the $B_{4}=S^{2} \times T^{2}$ solutions. After dimensional reduction and $T$-duality the Killing vector $\partial_{\psi}$ does not become the Reeb Killing vector of the Sasaki-Einstein space itself (which is known to be dual to the $R$-symmetry) but rather a linear combination of the Reeb vector with another Killing vector. The gauge transformations of $C_{(3)}$ account for the latter Killing vector. In fact, we can be more explicit and show that if we dimensionally reduce our ansatz above then we recover the ansatz for the consistent truncation of the $A d S_{5} \times Y^{p, q}$ solutions to metric plus $R$-symmetry gauge field that was discussed in [16]. This is carried out in appendix C.

We now want to calculate the $R$-charges of the chiral primaries dual to the wrapped membranes that we considered in section 3. In the Lagrangian for a membrane wrapping the supersymmetric cycle $\Sigma_{2}$, the Wess-Zumino term gives rise to the term

$$
\tau_{M 2} \int_{\Sigma_{2}} C_{(3)}=\frac{1}{2} R A .
$$

In analogy with the way in which a classical Lagrangian particle of charge $q$ travelling in an electromagnetic field couples through a four-velocity term $q A_{\mu} v^{\mu}$, we deduce that the wrapped branes carry $R$ units of $R$-charge. The factor of $1 / 2$ appearing in (4.10) comes from properly normalising the gauge field as we discuss in appendix C. In particular $A / 2$ is the natural normalisation for the gauge field.

Now consider the expression for $C_{(3)}$ in the consistent truncation ansatz (4.3), (4.4). Focussing on the terms that are linear in $A$ we get

$$
\begin{aligned}
\tau_{M 2} \int_{\Sigma_{2}} C_{(3)} & =\frac{1}{3} A \tau_{M 2} L^{3} \int_{\Sigma_{2}} e^{3 \lambda} Y^{\prime} \\
& =\frac{1}{3} A \tau_{M 2} L^{3} \int_{\Sigma_{2}} e^{3 \lambda} v_{M_{6}}
\end{aligned}
$$

where we used the calibration condition (3.5). We thus deduce that the $R$-charge of the supersymmetric wrapped membranes are given by

$$
R=\frac{2}{3} \tau_{M 2} L^{3} \int_{\Sigma_{2}} e^{3 \lambda} \operatorname{vol}_{M_{6}} .
$$

Since for chiral primaries we have $\Delta=\frac{3}{2} R$, this provides a derivation of our formula for $\Delta$ given in (3.1). 


\subsection{Thermal Plasma}

The consistency of our Kaluza-Klein truncation implies, by definition, that any solution of minimal gauged supergravity can be uplifted to eleven dimensions. In particular, black hole solutions of minimal gauged supergravity with non-vanishing $R$-charge can be uplifted and this allows us to extract non-trivial information about the hydrodynamics of the strongly coupled thermal plasma of the dual field theory.

In fact the relevant calculations were all carried out in detail in [16] (these calculations were extended from minimal gauged supergravity to a more general class of theories in [27]). One first extracts the thermodynamical quantities of the black hole solutions using the technique of [28]. To study the hydrodynamics of the thermal plasma dual to the black holes, one needs to calculate the retarded Green's function of the boundary stress tensor using the prescription of [29]. The shear viscosity $\eta$ can then be obtained from a Kubo relation.

Using this analysis we conclude that for the most general supersymmetric $A d S_{5}$ solutions of M-theory, the thermal plasmas of the dual CFTs with non-zero $R$-charge chemical potential all have the property that

$$
\frac{\eta}{s}=\frac{1}{4 \pi}
$$

In addition, again using the results of [16], we can conclude that the speed of sound in the thermal plasma is $1 / \sqrt{3}$.

\section{Discussion}

In this paper we have obtained some results about the CFTs dual to supersymmetric $A d S_{5}$ solutions that were analysed in [1]. In section 2, for some explicit solutions, we explicitly calculated the central charges of the dual CFTs. For the solutions with $B_{4}=K E_{4}$ or $B_{4}=S^{2} \times S^{2}$ the calculations were performed in the special case that $c=0$. It would be interesting to extend this to $c \neq 0$ but repeating the same steps is messy since the range of the coordinate $y$ is then given by the roots of a quartic, rather than, effectively, a quadratic.

In section 3, for the most general class of $A d S_{5}$ solutions of [1] we found the conditions for wrapped membranes to be supersymmetric by elucidating the appropriate generalised calibration two-form which can be constructed from the Killing spinors. For the explicit solutions we showed that various two-cycles are supersymmetric and then calculated the conformal dimensions of the corresponding chiral primaries. 
In section 4 we showed that for the most general solutions of [1] there is a consistent Kaluza-Klein truncation to minimal gauged supergravity in five dimensions. The gauge field corresponds to the abelian $R$-symmetry of the $N=1 \mathrm{SCFT}$. We used the consistent truncation to determine some properties of the hydrodynamics of the dual CFT using the results of [16]. In particular, we extended the domain of validity of the universal result that $\eta / s=1 /(4 \pi)$.

The fact that the Kaluza-Klein reduction is a consistent truncation is somewhat surprising. For cases in which the solutions have additional isometries, we do not expect, in general, to be able to extend the result to include more gauge fields (e.g. see [17]). However, for the special class of solutions with $N=2$ supersymmetry contained in [1] (and further analysed in [30]) it seems plausible that there is a consistent truncation that maintains the whole $S U(2) \times U(1) R$-symmetry. More generally it is natural to conjecture that the most general supersymmetric $A d S_{n}$ solutions always admit consistent truncations to a gauged supergravity that contain at least some if not all of the $R$-symmetry. It would be interesting to investigate this conjecture using the classification results of [36].

\section{Acknowledgements}

We would like to thank Alex Buchel and Dan Waldram for helpful discussions. JPG is supported by an EPSRC Senior Fellowship and a Royal Society Wolfson Award. JPG would also like to thank the Aspen Center for Physics where some of this work was done. OV wishes to thank the Generalitat Valenciana for the FPI research fellowship that he enjoyed whilst some of this work was carried out, and Imperial College for its warm hospitality during his visit at the early stages of this work.

\section{A $\kappa$-symmetry}

The condition for a probe membrane to be supersymmetric is that it satisfies the condition 31

$$
\Gamma_{\kappa} \epsilon=\epsilon,
$$

Here $\epsilon$ is a $D=11$ Killing spinor and the $\kappa$-symmetry projection matrix $\Gamma_{\kappa}$ is given by

$$
\Gamma_{\kappa}=\frac{1}{3 ! \sqrt{-g}} \epsilon^{u_{1} u_{2} u_{3}} \sigma_{u_{1} u_{2} u_{3}}
$$


where here $g$ is the determinant of the induced world-volume metric and $\sigma_{u}$ are induced world-volume gamma matrices: if the membrane is defined by the maps $x^{\mu}(\xi)$, we have $\sigma_{u}=\partial_{u} x^{\mu} \Gamma_{\mu}$ where $\Gamma^{\mu}$ are the $D=11$ Gamma matrices.

For the general $D=11$ solutions of [1] the $D=11$ Killing spinors take the form

$$
\epsilon=\psi \otimes e^{\lambda / 2} \xi
$$

where $\psi$ is a Killing spinor on $A d S_{5}$ and $\xi$ is a spinor on $M_{6}$ satisfying differential equations written in [1]. The $D=11$ gamma matrices can be decomposed as

$$
\begin{aligned}
\Gamma^{a} & =\rho^{a} \otimes \gamma_{7}, \\
\Gamma^{m} & =1 \otimes \gamma^{m},
\end{aligned}
$$

where $a, b=0,1, \ldots, 4$ and $m, n=1,2, \ldots, 6$ are frame indices on $A d S_{5}$ and $M_{6}$ respectively, and we have

$$
\left[\rho^{a}, \rho^{b}\right]_{+}=-2 \eta^{a b}, \quad\left[\gamma^{m}, \gamma^{n}\right]_{+}=2 \delta^{m n}
$$

with $\eta^{a b}=\operatorname{diag}(-1,1,1,1,1)$ and $\rho_{01234}=-1$.

It will be useful to have an explicit expression for the Killing spinors $\psi$. In global co-ordinates the metric on $A d S_{5}$ can be written as

$$
d s^{2}\left(A d S_{5}\right)=\left[-\cosh ^{2} r d t^{2}+d r^{2}+\sinh ^{2} r d \Omega_{3}^{2}\right]
$$

where

$$
d \Omega_{3}^{2}=\left(d \alpha_{1}\right)^{2}+\sin ^{2} \alpha_{1}\left[\left(d \alpha_{2}\right)^{2}+\sin ^{2} \alpha_{2}\left(d \alpha_{3}\right)^{2}\right],
$$

is the metric of a unit three-sphere parametrised by the three angles $\left(\alpha_{1}, \alpha_{2}, \alpha_{3}\right)$, with $0 \leq \alpha_{1}, \alpha_{2} \leq \pi$ and $0 \leq \alpha_{3} \leq 2 \pi$. The Killing spinors satisfy

$$
D_{a} \psi \equiv\left(\partial_{a}-\frac{1}{4} \omega_{a b c} \rho^{b c}\right) \psi=\frac{i}{2} \rho_{a} \psi
$$

and, in the obvious orthonormal frame, take the explicit form (see [32, 33])

$$
\psi=e^{i \frac{r}{2} \rho_{1}} e^{i \frac{t}{2} \rho_{0}} e^{\frac{\alpha_{1}}{2} \rho_{21}} e^{\frac{\alpha_{2}}{2} \rho_{32}} e^{\frac{\alpha_{3}}{2} \rho_{43}} \psi_{0}
$$

where $\psi_{0}$ is a constant spinor on $A d S_{5}$.

It will also be useful to recall [1] that the spinor $\xi$ on $M_{6}$ can be written

$$
\xi=\sqrt{2} \cos \alpha \eta_{1}+\sqrt{2} \sin \alpha \eta_{2}^{*}
$$


where $\zeta=\pi / 2-2 \alpha$, and $\eta_{1}$ and $\eta_{2}^{*}$ are unit norm chiral spinors on the $M_{6}$ satisfying the projections

$$
\begin{aligned}
& \gamma_{12} \eta_{1}=\gamma_{34} \eta_{1}=-\gamma_{56} \eta_{1}=i \eta_{1}, \\
& \gamma_{12} \eta_{2}^{*}=\gamma_{34} \eta_{2}^{*}=\gamma_{56} \eta_{2}^{*}=i \eta_{2}^{*} .
\end{aligned}
$$

Here the components $1,2,3,4$ refer to the base of the $S U(2)$ structure defined by $\xi$ and 5, 6 refer to the other two directions: see (2.2), (2.3) (and we refer to [1] for more details).

We are now in a position to show that the membranes wrapping the two-cycles calibrated by $Y^{\prime}$ that were considered in the text are in indeed supersymmetric. For simplicity we only present the details for the solutions with $B_{4}=K E_{4}$.

Case 1 : Fibre membrane probe. We first consider a probe membrane wrapping the $S^{2}$ fibre parametrised by $y, \psi$. We therefore simply set $\xi^{u}=(t, y, \psi)$. Using this embedding and decomposing the $D=11$ gamma-matrices we find that $\Gamma_{\kappa}$ reduces to:

$$
\Gamma_{\kappa}=\rho_{0} \otimes \gamma_{7} \gamma_{56}
$$

Since $\gamma_{7} \gamma_{56} \xi=\xi$ we deduce that the condition $\Gamma_{\kappa} \epsilon=\epsilon$ is equivalent to

$$
\rho_{0} \psi=\psi
$$

If we now return to the explicit expression for the $A d S_{5}$ spinor (A.9), we see that $\rho_{0}$ commutes with all matrices on the right hand side except for $\rho_{1}$. Thus we conclude that in order to preserve supersymmetry we must place our membrane probe at $r=0$, i.e. at the centre of $A d S_{5}$ space. Note that this condition arises because we have demanded that the wrapped membrane is independent of $t$.

Case 2 : Base membrane probe. We now consider membranes wrapping twocycles on the $\mathrm{KE}_{4}$ base. More precisely we show that a membrane wrapping a holomorphic cycle on $K E_{4}$ that is located at one of the poles of the fibration $y=y_{1}, y_{2}$ and at the centre of $A d S_{5}$ is supersymmetric. To see this we set $\xi^{u}=\left(t, \xi^{s}\right), s=1,2$, and consider configurations $x^{i}=x^{i}\left(\xi^{s}\right)$, where $x^{i}, i=1,2,3,4$ are coordinates on $K E_{4}$. We now find

$$
\Gamma_{\kappa}=\rho_{0} \otimes \frac{1}{\sqrt{g^{\prime}}}\left(\partial_{s} x^{i} \partial_{s} x^{j}\right) \gamma_{7} \gamma_{i j}
$$

where $g^{\prime}$ is the determinant of the induced metric on the two-cycle in $K E_{4}$. Suppose that we are sitting at the pole $y=y_{1}$ (the $y=y_{2}$ case is similar). We then have $\sin \zeta=-1$ corresponding to $\alpha=\pi / 2$ and hence $\gamma_{7} \gamma_{12} \xi=\gamma_{7} \gamma_{34} \xi=\xi$. If we again demand

$$
\rho_{0} \psi=\psi,
$$


which is achieved by locating the cycle at the centre of $A d S_{5}, r=0$, we see that the condition $\Gamma_{\kappa} \epsilon=\epsilon$ is precisely that for a supersymmetric two-cycle in $K E_{4}$. In particular, we must restrict to holomorphic curves in $K E_{4}$ in order to preserve supersymmetry.

\section{B Fermions and Consistent Truncation}

We now show that we can extend the consistent Kaluza-Klein truncation to include the fermions. We start with the variation of the $D=11$ gravitino $\Psi_{\mu}$ :

$$
\delta \Psi_{\mu}=\nabla_{\mu} \epsilon+\frac{1}{288}\left(\Gamma_{\mu}^{\nu_{1} \nu_{2} \nu_{3} \nu_{4}}-8 \delta_{\mu}^{\nu_{1}} \Gamma^{\nu_{1} \nu_{2} \nu_{3}}\right) G_{\nu_{1} \nu_{2} \nu_{3} \nu_{4}} \epsilon
$$

and decompose the $D=11$ Killing spinor as $\epsilon=\varepsilon \otimes e^{\lambda / 2} \xi$, where $\varepsilon$ is an arbitrary spinor on the external $D=5$ space-time and $\xi$ is the $M_{6}$ Killing spinor for the undeformed solutions. We will only need below the Kaluza-Klein ansatz for the external component of the gravitino; in tangent space, it reads $\Psi_{a}=\psi_{a} \otimes e^{-\lambda / 2} \xi$, where $\psi_{a}$ is the $D=5$ gravitino.

Combining this with the ansatz (4.1) for the metric and (4.5) for the four-form, and substituting into (B.1) we find that the components tangent to $M_{6}$,

$$
\begin{aligned}
& \delta \Psi_{i}=\delta^{0} \Psi_{i}+\frac{1}{144} F_{a b} \rho^{a b} \varepsilon \otimes\left(2 \gamma_{7} \gamma_{i 5} \cos \zeta+\left(\gamma_{i}{ }^{j k}-4 \delta_{i}^{j} \gamma^{k}\right) J_{j k} \sin \zeta-2 \gamma_{i 56}\right) e^{-\lambda / 2} \xi \\
& \delta \Psi_{5}=\delta^{0} \Psi_{5}+\frac{1}{144} F_{a b} \rho^{a b} \varepsilon \otimes\left(-4 \gamma_{7} \cos \zeta+\gamma_{5}{ }^{j k} J_{j k} \sin \zeta+4 \gamma_{6}\right) e^{-\lambda / 2} \xi \\
& \delta \Psi_{6}=\delta^{0} \Psi_{6}+\frac{1}{144} F_{a b} \rho^{a b} \varepsilon \otimes\left(2\left(3-\gamma_{7} \gamma_{56}\right) \cos \zeta+\gamma_{6}{ }^{j k} J_{j k} \sin \zeta-4 \gamma_{5}\right) e^{-\lambda / 2} \xi
\end{aligned}
$$

vanish identically. Indeed, in $(\overline{\mathrm{B} .2}), \delta^{0}$ corresponds to the variation of the undeformed gravitino, which vanishes due to the fact that $\xi$ is the Killing spinor of the undeformed solutions, and the $F$-dependent terms also vanish on account of the projections satisfied by $\xi$ that can be read off from (A.11), (A.12).

For the external directions $\delta \Psi_{a}$ of (B.1) we find, once the projections on $\xi$ have been taken into account,

$$
\begin{aligned}
\delta \psi_{a} & \otimes e^{-\lambda / 2} \xi=\left(\nabla_{a}-A_{a} \partial_{\psi}-\frac{1}{24} F_{b c}\left(\rho_{a}^{b c}+4 \delta_{a}^{b} \rho^{c}\right)-\frac{i}{2} \rho_{a}\right) \varepsilon \otimes e^{-\lambda / 2} \xi \\
+ & \frac{1}{2} \rho_{a} \varepsilon \otimes\left(i+\partial_{m} \lambda \gamma_{7} \gamma^{m}+\frac{1}{144} e^{-3 \lambda} \gamma_{7} \gamma^{m_{1} m_{2} m_{3} m_{4}} g_{m_{1} m_{2} m_{3} m_{4}}\right) e^{-\lambda / 2} \xi
\end{aligned}
$$

Using the fact that $\xi=e^{\frac{i}{2} \psi} \xi_{0}$, where $\xi_{0}$ is independent of the coordinate 2 , and

\footnotetext{
${ }^{2}$ This follows from equations (2.45), (2.32), (C.13), (B.6), (B.1) of [1] and the fact that $\lambda$ is $\psi$-independent. Here, the $D=5$ gravitino $\psi_{a}$ should not be confused with the $M_{6}$ coordinate $\psi$.
} 
equation (2.8) of [1], this becomes

$$
\delta \psi_{a}=\left(\nabla_{a}-\frac{i}{2} A_{a}-\frac{1}{24}\left(\rho_{a}^{b c}+4 \delta_{a}^{b} \rho^{c}\right) F_{b c}-\frac{i}{2} \rho_{a}\right) \varepsilon .
$$

This is precisely the supersymmetry variation of the gravitino in minimal $D=5$ gauged supergravity corresponding to the Lagrangian whose bosonic terms are as in (4.9).

\section{Consistent Truncation and Normalisation of $R$ - Charge}

For the explicit solution with $B_{4}=S^{2} \times T^{2}$, the Kaluza-Klein ansatz (4.2), (4.3), (4.4) takes the detailed form

$$
d s^{2}\left(M_{6}\right)=e^{-6 \lambda} d s^{2}\left(T^{2}\right)+\frac{1-c y}{6} d s^{2}\left(S^{2}\right)+e^{-6 \lambda} \sec ^{2} \zeta d y^{2}+\frac{1}{9} \cos ^{2} \zeta(D \psi+A)^{2},
$$

and, setting $L=1$ here for simplicity,

$$
\begin{aligned}
G_{(4)}=h_{3} \operatorname{vol}\left(S^{2}\right) & \operatorname{vol}\left(T^{2}\right)+\left[h_{1} \operatorname{vol}\left(S^{2}\right)+h_{2} \operatorname{vol}\left(T^{2}\right)\right] d y(D \psi+A) \\
& +\left[\alpha_{1} \operatorname{vol}\left(S^{2}\right)+\alpha_{2} \operatorname{vol}\left(T^{2}\right)+\frac{1}{9} d y(D \psi+A)\right] F+\frac{1}{3} d y\left(*_{5} F\right),
\end{aligned}
$$

where $h_{i}=h_{i}(y)$ are the functions appearing in the undeformed flux (2.33) and

$$
\begin{aligned}
\alpha_{1} & =\frac{(c y-1) y}{9}, \\
\alpha_{2} & =\frac{(c y-1) y}{3\left(a-y^{2}\right)} .
\end{aligned}
$$

This can be dimensionally reduced and $T$-dualised (e.g. using the formula contained in appendix $\mathrm{C}$ of [19]) to obtain a Kaluza-Klein ansatz for the $A d S_{5} \times Y^{p, q}$ solutions of type IIB. We find:

$$
\begin{aligned}
d s^{2} & =d s_{5}^{2}+\frac{1-c y}{6} d s^{2}\left(S^{2}\right)+e^{-6 \lambda} \sec ^{2} \zeta d y^{2}+\frac{1}{9} \cos ^{2} \zeta(D \psi+A)^{2} \\
& +e^{6 \lambda}\left[d \alpha-\frac{c}{6} A+\frac{-2 y+y^{2} c+a c}{6\left(a-y^{2}\right)}(D \psi+A)\right]^{2}, \\
F_{5} & =-4 v^{2} l_{5}+\left(d \alpha-\frac{c}{6} A\right)\left[-\frac{2(1-c y)}{9} \operatorname{vol}\left(S^{2}\right) d y(D \psi+A)+\frac{(c y-1) y}{9} \operatorname{vol}\left(S^{2}\right) F\right. \\
& \left.+\frac{1}{9} d y(D \psi+A) F+\frac{1}{3} d y\left(*_{5} F\right)\right]+\frac{1-c y}{18} \operatorname{vol}\left(S^{2}\right)\left(*_{5} F\right)+\frac{c}{18} d y\left(*_{5}\right) F(D \psi+A) \\
& +\frac{(c y-1)^{2}}{54} \operatorname{vol}\left(S^{2}\right) F(D \psi+A) .
\end{aligned}
$$


In this form we clearly see that we have gauged shifts of the $\psi$ coordinate as well as the $\alpha$ co-ordinate (when $c$ is non-zero). In order to make the Reeb vector manifest, we can employ the coordinate change $\alpha=-\beta / 6-c \psi^{\prime} / 6, \psi=\psi^{\prime}$ to get

$$
\begin{aligned}
d s^{2} & =d s_{5}^{2}+\frac{1-c y}{6} d s^{2}\left(S^{2}\right)+e^{-6 \lambda} \sec ^{2} \zeta d y^{2}+\frac{1}{36} e^{6 \lambda} \cos ^{2} \zeta(d \beta+c \cos \theta d \phi)^{2} \\
& +\frac{1}{9}\left(d \psi^{\prime}+\mathcal{A}+A\right)^{2} \\
F_{5} & =-4 v o l_{5}+\frac{1}{3} j\left(*_{5} F\right)-\frac{4}{3} v o_{4}\left(d \psi^{\prime}+\mathcal{A}+A\right)+\frac{1}{9} j\left(d \psi^{\prime}+\mathcal{A}+A\right) F,
\end{aligned}
$$

where $\theta, \phi$ parametrise $S^{2}$, and $j$, the Kähler-form on the locally Kähler-Einstein base space of the Sasaki-Einstein space $Y^{p, q}$ orthogonal to the orbits of the Reeb vector $\partial_{\psi^{\prime}}$, is given by

$$
j=\frac{1-c y}{6} \operatorname{vol}\left(S^{2}\right)+\frac{1}{6} d y(d \beta+c \cos \theta d \phi) .
$$

In addition, $v o l_{4}$ is the volume form of the Kähler base, $v o l_{4}=\frac{1}{2} j^{2}$, and the potential $\mathcal{A}$, given by $\mathcal{A}=-\cos \theta d \phi+y(d \beta+c \cos \theta d \phi)$ satisfies $d \mathcal{A}=6 j$. This is precisely the ansatz discussed in [16]. In particular we see that we have gauged shifts of the coordinate $\psi^{\prime}$ associated with the Reeb vector, which is known to be dual to the $R$-symmetry. This provides confirmation that the $M$-theory consistent truncation is indeed a reduction maintaining the $R$-charge gauge field.

Let us now return to the consistent truncation for the most general solutions and determine the correct normalisation of the gauge field. The consistent truncation ansatz that we considered is invariant under the gauge transformations

$$
\begin{aligned}
& \psi \rightarrow \psi-\epsilon, \\
& A \rightarrow A+d \epsilon .
\end{aligned}
$$

To normalise the gauge field, following [20], we look for an object with known $R$ charge. To do this we first think of our $A d S_{5} \times M_{6}$ solutions as special cases of solutions of the form $M^{1,3} \times M_{7}$ where $M^{1,3}$ is four-dimensional Minkowski space. As discussed in [1] the relevant $M_{7}$ has a (local) $S U(3)$ structure given by a vector $K^{\prime}$, a three-form $\Omega^{\prime}$ and a two-form $J^{\prime}$ (see [34]). It was shown in [35] that such solutions give rise to a superpotential of the form

$$
W \sim \int_{M_{7}}\left(G_{(4)}+i K^{\prime} d J^{\prime}\right) \Omega^{\prime},
$$

Since the superpotential has $R$-charge 2 , we conclude that $\Omega^{\prime}$ has $R$-charge 2 . Thus we just need to relate $\Omega^{\prime}$ to the $S U(2)$ structure on $M_{6}$. In fact this was explicitly done in [1]:

$$
\Omega^{\prime}=e^{-3 \lambda} \hat{\Omega}\left(-\sin \zeta K_{1}-\cos \zeta d r+i K_{2}\right) .
$$


The analysis of [1] shows that the important $\psi$ dependence only appears in the two-

form $\hat{\Omega}$ and is of the form $\hat{\Omega} \sim e^{i \psi}$. We thus conclude that $\psi / 2$ is the appropriately normalised coordinate and hence that $A / 2$ is the appropriately normalised gauge field.

\section{References}

[1] J. P. Gauntlett, D. Martelli, J. Sparks and D. Waldram, "Supersymmetric $\operatorname{AdS}(5)$ solutions of M-theory," Class. Quant. Grav. 21 (2004) 4335 arXiv:hep-th/0402153.

[2] J. P. Gauntlett, D. Martelli, J. Sparks and D. Waldram, "Sasaki-Einstein metrics on S(2) x S(3)," Adv. Theor. Math. Phys. 8 (2004) 711 [arXiv:hep-th/0403002].

[3] S. Benvenuti, S. Franco, A. Hanany, D. Martelli and J. Sparks, "An infinite family of superconformal quiver gauge theories with Sasaki-Einstein duals," JHEP 0506 (2005) 064 arXiv:hep-th/0411264.

[4] I. R. Klebanov and A. A. Tseytlin, "Entropy of Near-Extremal Black p-branes," Nucl. Phys. B 475 (1996) 164 [arXiv:hep-th/9604089].

[5] J. Gutowski, G. Papadopoulos and P. K. Townsend, "Supersymmetry and generalized calibrations," Phys. Rev. D 60 (1999) 106006 [arXiv:hep-th/9905156].

[6] G. Policastro, D. T. Son and A. O. Starinets, "The shear viscosity of strongly coupled N = 4 supersymmetric Yang-Mills Phys. Rev. Lett. 87 (2001) 081601 arXiv:hep-th/0104066.

[7] G. Policastro, D. T. Son and A. O. Starinets, JHEP 0209 (2002) 043 arXiv:hep-th/0205052.

[8] C. P. Herzog, "The hydrodynamics of M-theory," JHEP 0212 (2002) 026 arXiv:hep-th/0210126.

[9] P. Kovtun, D. T. Son and A. O. Starinets, "Holography and hydrodynamica: Diffusion on stretched horizons," JHEP 0310 (2003) 064 arXiv:hep-th/0309213].

[10] A. Buchel and J. T. Liu, "Universality of the shear viscosity in supergravity," Phys. Rev. Lett. 93 (2004) 090602 arXiv:hep-th/0311175. 
[11] P. Kovtun, D. T. Son and A. O. Starinets, "Viscosity in strongly interacting quantum field theories from black hole physics," Phys. Rev. Lett. 94 (2005) 111601 arXiv:hep-th/0405231.

[12] J. Mas, "Shear viscosity from R-charged AdS black holes," JHEP 0603 (2006) 016 arXiv:hep-th/0601144].

[13] D. T. Son and A. O. Starinets, "Hydrodynamics of R-charged black holes," JHEP 0603 (2006) 052 arXiv:hep-th/0601157].

[14] K. Maeda, M. Natsuume and T. Okamura, "Viscosity of gauge theory plasma with a chemical potential from AdS/CFT," Phys. Rev. D 73 (2006) 066013 arXiv:hep-th/0602010|.

[15] O. Saremi, "The viscosity bound conjecture and hydrodynamics of M2-brane theory at arXiv:hep-th/0601159.

[16] A. Buchel and J. T. Liu, "Gauged supergravity from type IIB string theory on $Y^{p, q}$ manifolds," arXiv:hep-th/0608002.

[17] P. Hoxha, R. R. Martinez-Acosta and C. N. Pope, "Kaluza-Klein consistency, Killing vectors, and Kaehler spaces," Class. Quant. Grav. 17 (2000) 4207 arXiv:hep-th/0005172.

[18] M. Henningson and K. Skenderis, "The Holographic Weyl anomaly", JHEP 9807 (1998) 023, hep-th/9806087.

[19] J. P. Gauntlett, O. A. P. Mac Conamhna, T. Mateos and D. Waldram, "New supersymmetric AdS(3) solutions," arXiv:hep-th/0608055.

[20] D. Berenstein, C. P. Herzog and I. K. Klebanov, "Baryon spectra and AdS/CFT correspondence", JHEP 06 (2002) 047, hep-th/0202150.

[21] J. P. Gauntlett and S. Pakis, "The geometry of D $=11$ Killing spinors," JHEP 0304 (2003) 039 [arXiv:hep-th/0212008].

[22] D. Arean, D. E. Crooks and A. V. Ramallo, "Supersymmetric probes on the conifold," JHEP 0411 (2004) 035 arXiv:hep-th/0408210].

[23] F. Canoura, J. D. Edelstein, L. A. P. Zayas, A. V. Ramallo and D. Vaman, "Supersymmetric branes on $\operatorname{AdS}(5) \times Y^{p, q}$ and their field theory duals," JHEP 0603 (2006) 101 [arXiv:hep-th/0512087]. 
[24] C. P. Herzog, Q. J. Ejaz and I. R. Klebanov, "Cascading RG Flows from New Sasaki-Einstein Manifolds", JHEP 0502 (2005) 009, hep-th/0412193.

[25] D. Martelli and J. Sparks, "Toric geometry, Sasaki-Einstein manifolds and a new infinite class of AdS/CFT duals", Commun.Math.Phys.262 (2006) 51-89, hep-th/0411238.

[26] S. Benvenuti and M. Kruczenski, "Semiclassical strings in Sasaki-Einstein manifolds and long operators in $\mathrm{N}=1$ gauge theories," JHEP 0610 (2006) 051 arXiv:hep-th/0505046.

[27] P. Benincasa, A. Buchel and R. Naryshkin, "The shear viscosity of gauge theory plasma with chemical potentials," arXiv:hep-th/0610145.

[28] V. Balasubramanian and P. Kraus, "A stress tensor for anti-de Sitter gravity," Commun. Math. Phys. 208 (1999) 413 [arXiv:hep-th/9902121].

[29] D. T. Son and A. O. Starinets, "Minkowski-space correlators in AdS/CFT correspondence: Recipe and applications," JHEP 0209 (2002) 042 arXiv:hep-th/0205051.

[30] H. Lin, O. Lunin and J. M. Maldacena, "Bubbling AdS space and 1/2 BPS geometries," JHEP 0410 (2004) 025 [arXiv:hep-th/0409174].

[31] K. Becker, M. Becker and A. Strominger, "Five-Branes, Membranes And Nonperturbative String Theory," Nucl. Phys. B 456 (1995) 130 arXiv:hep-th/9507158.

[32] H. Lu, C. N. Pope and J. Rahmfeld, "A construction of Killing spinors on $S^{n}$," J. Math. Phys. 40 (1999) 4518 arXiv:hep-th/9805151.

[33] M. T. Grisaru, R. C. Myers and O. Tafjord, "SUSY and Goliath," JHEP 0008 (2000) 040 arXiv:hep-th/0008015.

[34] P. Kaste, R. Minasian and A. Tomasiello, "Supersymmetric M-theory compactifications with fluxes on seven-manifolds and G-structures", JHEP 0307 (2003) 004, hep-th/0303127.

[35] K. Behrndt and C. Jeschek, "Superpotentials from flux compactifications of Mtheory," Class. Quant. Grav. 21 (2004) S1533 arXiv:hep-th/0401019. 
[36] J. P. Gauntlett, O. A. P. Mac Conamhna, T. Mateos and D. Waldram, "AdS spacetimes from wrapped M5 branes," arXiv:hep-th/0605146. 